\title{
A Passivity-Based Approach for Trajectory Tracking and Link-Side Damping of Compliantly Actuated Robots
}

\author{
Manuel Keppler ${ }^{1}$, Dominic Lakatos ${ }^{1}$, Christian Ott ${ }^{1}$, Alin Albu-Schäffer ${ }^{2}$
}

\begin{abstract}
This paper presents a control method to implement trajectory tracking and disturbance rejection characteristics for the link-side dynamics of compliantly actuated robots with nonlinear spring characteristics. This is achieved by introducing new motor coordinates reflecting the damping and feedforward terms and shaping the dynamics of the motor such that it structurally equals the dynamics in the original coordinates. Thus, the approach achieves the control goal while changing the original plant dynamics only to a minimum extent. Passivity, stability, and convergence properties of the closedloop dynamics are proven. The performance of the control approach has been experimentally evaluated on the variable stiffness robot arm DLR Hand Arm System, where the stiffness in each of the joints is highly nonlinear. To our best knowledge, this is the first experimentally validated tracking controller for compliantly actuated robots with nonlinear elastic elements.
\end{abstract}

\section{INTRODUCTION}

To handle impacts and unknown contact forces, robot design recently evolved from rigid towards compliant actuators having real, generally nonlinear springs in their power train [1]. The elastic elements acting between motor and link inertias together with the intrinsic damping properties of any physical system lead to a dynamical behavior of a low-pass filter on external loads. As a consequence high force peaks resulting, for instance, from unexpected environmental collisions act attenuated on gear box and motor parts preventing the destruction of these parts. Therefore, the elastic elements can significantly increase the mechanical robustness on the one side, but they also introduce oscillatory dynamics [2] into the plant on the other side. Especially, if the contact impedance matches according to requirements of mechanical robustness, resulting frequencies of the oscillatory dynamics are in a range which is undesired for accurate link motion tracking control. To benefit from the mechanical robustness properties of compliantly actuated robots while simultaneously achieving a desired tracking dynamics of the manipulator links, damping and the required feedforward terms have to be added to the system by control.

Solutions to the regulation problem of link configuration variables have been proposed in [3], [4], [5], and [6]. These methods are exclusively introduced for so-called flexible joint robots (FJR), where elasticities in links and power trains are modelled as linear springs (i. e., constant stiffness), concentrated in the joints. A generalization to the case of nonlinear elasticities as appearing in many implementations

\footnotetext{
${ }^{1}$ The authors are with the Institute of Robotics and Mechatronics, German Aerospace Center (DLR), D-82234 Oberpfaffenhofen, Germany. Contact: manuel . kepplerdalr.de

${ }^{2}$ Alin Albu-Schäffer is also with Technical University Munich, Chair of Sensor Based Robots and Intelligent Assistance Systems, Department of Informatics, D-85748 Garching, Germany.
}

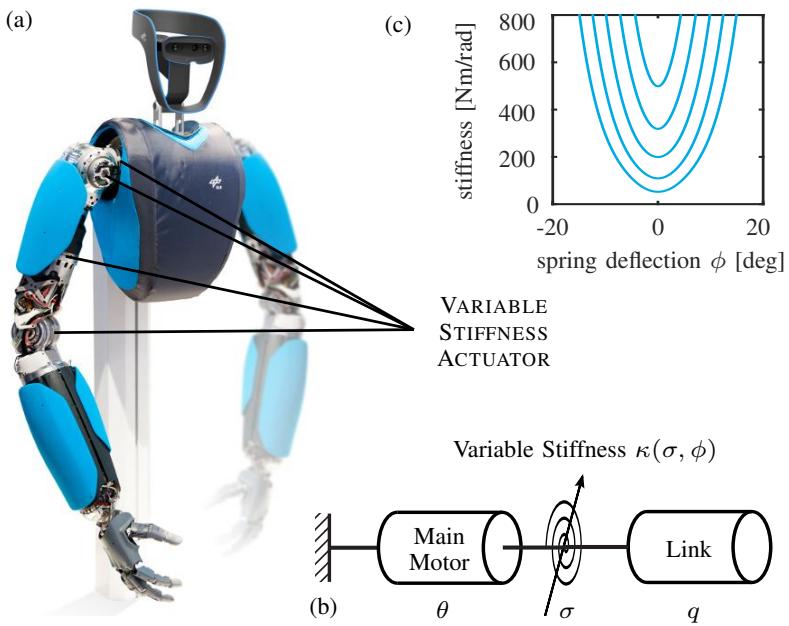

Fig. 1. Fig. 1a shows the DLR Hand Arm System. Fig. 1b shows a simplified working scheme of a single VSA. Fig. 1c depicts the stiffness characteristics for stiffness adjuster positions $\sigma=\left[\begin{array}{lll}0 & 2.5 & 5.0 \\ 7.5 & 10\end{array}\right] \mathrm{deg}$, the outer curve corresponds to $\sigma=0 \mathrm{deg}$.

of variable stiffness actuators (VSA) [7] has been proposed in [8]. As all above methods consider only collocated feedback $^{1}$, the convergence performance depends strongly on the joint stiffness, i.e., low stiffness required for impact attenuation leads to low damping performance. Regulation controller with high damping performance considering also feedback of non-collocated variables are reported in [9], [10], [11]. While the former approach [9] (applicable to FJR) provides a comprehensive stability analysis, the latter methods [10], [11] (applicable to VSA robots) are not proven to be stable yet. The three latter controllers are link-side damping controllers for the regulation case which are of course of less performance if the set-point is not constant.

Solutions to the tracking problem of robots with elastic transmissions are reported in the pioneering works [12], [13], [14]. Further relevant tracking controllers are based on cascaded structures [15], integrator backstepping [16], [17, Chap. 6.2], extensions of the well known Slotine and Li controller [18] to the flexible joint case [19], feedback linearization [12], [20], [21], and integral manifold control [12]. The above mentioned papers either derive their results only for the FJR case (except the method in [21] which applies to VSA robots) or validate their controllers only in computer simulations (except in [15] experimental results of a single FJR joint). The same is true for the comprehensive

${ }^{1}$ Control input collocated variables, are variables for which its time derivatives form a conjugate power pair together with the control input (torque). 
ORIGINAL Plant DYNAMics

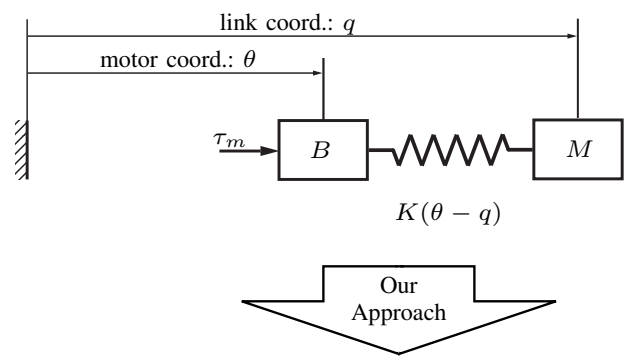

ACHieved Closed Loop Dynamics

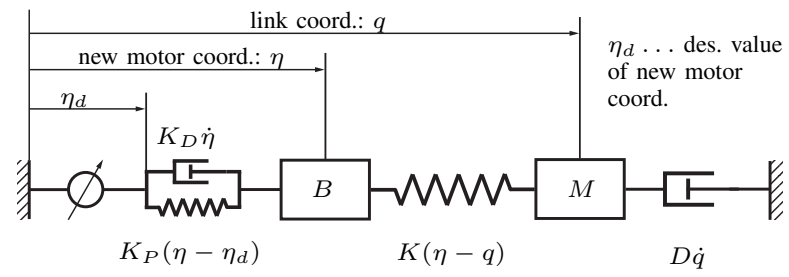

Fig. 2. Design idea shown for the simplest form of a single joint with flexible transmission: adding feedback control $\boldsymbol{\tau}_{m}$ which adds stability and link-side damping while changing the original dynamics only to a minimal extent.

overview given in [22] and the supplementing survey given by [23].

This paper aims at closing this gap by solving the tracking problem for multi-joint robots with nonlinear elastic transmission (see Fig. 1) from a theoretical (stability analysis) and a practical (experimental validation) point of view. The idea is to exclusively add the damping and feedforward terms to the dynamics of the link variables without changing the rigid body dynamics of the links nor the structure of the nonlinear springs. This is achieved by introducing new coordinates reflecting these damping and feedforward terms and then expressing the motor dynamics in the new coordinates while shaping the resulting dynamics such that it structurally equals the dynamics of the original coordinates. The desired tracking behavior (and disturbance rejection) of the link coordinates resulting from the original link dynamics with the additional desired damping and feedforward terms are achieved by pure PD regulation control in the new coordinates. Since the controlled motor dynamics and the dynamics of the link variables can be represented as an interconnection of passive subsystems, a comprehensive stability statement can be concluded based on physically motivated Lyapunov functions. In contrast to the state of the art methods mentioned above, this controller achieves the exact tracking and damping behavior by changing the original plant dynamics only to a minimum extent. To our best knowledge, this is the first link-side tracking controller of compliantly actuated multi-joint robots with nonlinear elastic transmissions which is theoretically founded (stability analysis) and experimentally verified to perform practically.

\section{DESIGN IDEA}

Consider the simplest form of a robot joint with flexible transmission as depicted in Fig. 2a. The motor inertia $B$ acts via a spring $f=K(\theta-q)$ with stiffness $K$ on the link inertia $M$, where $\theta$ and $q$ are motor and link coordinates respectively. The control input is a force $\tau_{m}$ acting on the motor inertia $B$. This results in dynamics of the form:

$$
\begin{aligned}
M \ddot{q} & =K(\theta-q) \\
B \ddot{\theta}+K(\theta-q) & =\tau_{m}
\end{aligned}
$$

Since the elasticity is introduced to exploit the energy storing capabilities, compliant actuators are often designed such that damping and friction in parallel to the spring is negligible. As such mechanical robustness against impacts and unknown contact forces is achieved at one side, but also intrinsic oscillatory dynamics result on the other side. The goal is to find a feedback control $\tau_{m}$ which exclusively adds stability and a damping term $D \dot{q}$ by changing the remaining dynamics structurally only to a minimum extent. This is achieved by introducing a new motor coordinate $\eta$ satisfying

$$
K(\theta-q)=K(\eta-q)-D \dot{q}
$$

which implements the desired damping term. It can be easily verified that the control law

$$
\tau_{m}=\bar{u}-B K^{-1} D q^{(3)}-D \dot{q}
$$

results in the dynamics

$$
\begin{aligned}
M \ddot{q} & =K(\eta-q)-D \dot{q} \\
B \ddot{\eta}+K(\eta-q) & =\bar{u},
\end{aligned}
$$

which is visualized in Fig. 2. For simplicity we assume a constant damping gain D. Since the damping is already formulated in the coordinate transformation (3), the problem reduces to regulating $\eta$ to a desired value $\eta_{d}$, such that $q \rightarrow q_{d}$. This can be achieved using a simple PD control of the form

$$
\bar{u}=-K_{D} \dot{\eta}-K_{P}\left(\eta-\eta_{d}\right)
$$

which results in an entire closed-loop dynamics (see Fig. 2b)

$$
\begin{array}{r}
M \ddot{q}=K(\eta-q)-D \dot{q} \\
B \ddot{\eta}+K_{D} \dot{\eta}+K_{P}\left(\eta-\eta_{d}\right)+K(\eta-q)=0 .
\end{array}
$$

it can be easily verified that the only equilibrium point is $\eta=q, \eta=\eta_{d}$ and consequently $q=\eta_{d} \equiv q_{d}$.

The remainder of the paper applies these basic ideas to general robotic systems

- with nonlinear elastic transmissions,

- with multiple degrees of freedom,

- that are subject to gravity

and extends this control concept to

- the tracking case

\section{Problem Statement}

Consider the dynamics of a compliantly actuated robot satisfying

$$
\begin{aligned}
& \boldsymbol{M}(\boldsymbol{q}) \ddot{\boldsymbol{q}}+\boldsymbol{C}(\boldsymbol{q}, \dot{\boldsymbol{q}}) \dot{\boldsymbol{q}}=-\boldsymbol{g}(\boldsymbol{q})+\boldsymbol{\psi}(\boldsymbol{\theta}-\boldsymbol{q})+\boldsymbol{\tau}_{\text {ext }} \\
& \boldsymbol{B} \ddot{\boldsymbol{\theta}}+\boldsymbol{\psi}(\boldsymbol{\theta}-\boldsymbol{q})=\boldsymbol{\tau}_{m} .
\end{aligned}
$$


In the dynamics of the link-side (10), the inertia matrix $\boldsymbol{M}(\boldsymbol{q}) \in \mathbb{R}^{n \times n}$ is symmetric and positive definite, the Coriolis matrix $\boldsymbol{C}(\boldsymbol{q}, \dot{\boldsymbol{q}}) \in \mathbb{R}^{n \times n}$ is such that the matrix $\dot{\boldsymbol{M}}-2 \boldsymbol{C}$ is skew-symmetric, the vector of generalized gravitational forces $\boldsymbol{g}(\boldsymbol{q}) \in \mathbb{R}^{n \times n}$ is derived from the potential $U_{g}(\boldsymbol{q})$ such that

$$
\boldsymbol{g}(\boldsymbol{q}):=-\left(\frac{\partial U_{g}(\boldsymbol{q})}{\partial \boldsymbol{q}}\right)^{T}
$$

and $\tau_{\text {ext }} \in \mathbb{R}^{n}$ are generalized, externally applied forces. In the dynamics of the motors (11), the motor torque $\tau_{m}$ is the control input, the inertia matrix $\boldsymbol{B} \in \mathbb{R}^{n \times n}$ is assumed to be constant, symmetric and positive definite. The structure of compliantly actuated robots (10)-(11) represents an underactuated mechanical system in which only the generalized motor coordinates $\boldsymbol{\theta} \in \mathbb{R}^{n}$ can be directly actuated via the control input $\tau_{m} \in \mathbb{R}^{n}$. The generalized link coordinates $\boldsymbol{q} \in \mathbb{R}^{n}$ can only be indirectly actuated via the generalized elastic forces

$$
\boldsymbol{\psi}(\boldsymbol{\theta}-\boldsymbol{q}):=\left.\frac{\partial U_{s}(\boldsymbol{\phi})^{T}}{\partial \boldsymbol{\phi}}\right|_{\boldsymbol{\phi}=\boldsymbol{\theta}-\boldsymbol{q}} \in \mathbb{R}^{n},
$$

which are derived from the even, strictly convex and positive semi-definite potential function $U_{s}(\phi)$. This form of underactuation is the major challenge in the control of the states $\boldsymbol{q}, \dot{\boldsymbol{q}}$.

Due to the properties of $U_{s}(\phi), \boldsymbol{\psi}$ is a global diffeomorphism and thereby its inverse $f^{-1}$ always exists. We also assume

$$
U_{s}(\phi)=0 \Leftrightarrow \phi=\mathbf{0} .
$$

Remark 1: For VSA the elastic potential ${ }^{2}$ depends on additional variables $\boldsymbol{\sigma} \in \mathbb{R}^{n}$ which can be considered as an input to change the characteristics of the stiffness (see Fig. 1c), i.e. $U_{s}(\phi, \sigma)$, such that the generalized elastic force takes the form

$$
\boldsymbol{\psi}(\boldsymbol{\theta}-\boldsymbol{q}, \boldsymbol{\sigma}):=\left.\frac{\partial U_{s}(\boldsymbol{\phi}, \boldsymbol{\sigma})^{T}}{\partial \boldsymbol{\phi}}\right|_{\boldsymbol{\phi}=\boldsymbol{\theta}-\boldsymbol{q}} \in \mathbb{R}^{n} .
$$

This input can be altered to achieve a certain interaction behavior intrinsically (i.e., the characteristics of the real springs are changed). Since the focus of this work is on damping and tracking control, we consider $\boldsymbol{\sigma}$ as constant parameters. This reduces the complexity of the equations.

In the following, it is assumed that the potential function $U_{s}(\phi)$ is at least three times continuously differentiable. Further, it is assumed that for the total potential

$$
U(\boldsymbol{\theta}, \boldsymbol{q})=U_{g}(\boldsymbol{q})+U_{s}(\boldsymbol{q}-\boldsymbol{\theta})
$$

there exists constants $c_{1}, c_{2}, c_{3}, c_{4}, c_{5}>0$ such that the

\footnotetext{
${ }^{2}$ Condition (14) still holds true, i.e. $\forall \boldsymbol{\sigma} \in \mathbb{R}^{n}, U_{s}(\boldsymbol{\phi}, \boldsymbol{\sigma})=0 \Leftrightarrow \boldsymbol{\phi}=\mathbf{0}$.
}

inequalities

$$
\begin{aligned}
c_{1}\|\boldsymbol{q}\|^{2} & <\boldsymbol{q}^{T} \frac{\partial^{2} U(\boldsymbol{\theta}, \boldsymbol{q})}{\partial \boldsymbol{q}^{2}} \boldsymbol{q}<c_{2}\|\boldsymbol{q}\|^{2} \\
c_{3} & <\left\|\frac{\partial^{2} U(\boldsymbol{\theta}, \boldsymbol{q})}{\partial \boldsymbol{q} \partial \boldsymbol{\theta}}\right\|<c_{4} \\
c_{5}\|\boldsymbol{\theta}\|^{2} & <\boldsymbol{\theta}^{T} \frac{\partial^{2} U(\boldsymbol{\theta}, \boldsymbol{q})}{\partial \boldsymbol{\theta}^{2}} \boldsymbol{\theta}
\end{aligned}
$$

hold $^{3} \forall \boldsymbol{q}, \boldsymbol{\theta} \in \mathbb{R}^{n}$. These assumptions define the class of systems for which the control approach of this paper can be applied. Physical interpretations of these inequalities can be found in [8].

In this paper we address the problem of finding a control input $\tau_{m}$ which allows to influence the convergence behavior of $\dot{\boldsymbol{q}} \rightarrow \mathbf{0}$ via a link-side damping term $\boldsymbol{D} \dot{\boldsymbol{q}}$, satisfying $\dot{\boldsymbol{q}}^{T} \boldsymbol{D} \dot{\boldsymbol{q}}>0, \forall \dot{\boldsymbol{q}} \neq \mathbf{0}$, while simultaneously introducing link-side tracking behavior $\boldsymbol{q}(t) \rightarrow \boldsymbol{q}_{d}(t)$, where $\boldsymbol{q}_{d} \in \mathcal{C}^{4}$. Thereby, the goal is to change the original link dynamics (10) only to a minimum extent by keeping $\boldsymbol{M}(\boldsymbol{q}), \boldsymbol{C}(\boldsymbol{q}, \dot{\boldsymbol{q}})$, $\boldsymbol{g}(\boldsymbol{q})$, and the structure of $U_{s}(\boldsymbol{q}-\boldsymbol{\theta})$ unchanged and adding only damping and tracking terms.

\section{Controller Design}

\section{A. Desired Link-Side Dynamics}

We aim to find a controller $\tau_{m}$ in (11) such that the behavior of the resulting compensated system is similar to the following desired dynamics

$$
\begin{aligned}
\boldsymbol{M}(\boldsymbol{q}) \ddot{\tilde{\boldsymbol{q}}}+\boldsymbol{C}(\boldsymbol{q}, \dot{\boldsymbol{q}}) \dot{\tilde{\boldsymbol{q}}} & =-\boldsymbol{D} \dot{\tilde{\boldsymbol{q}}}+\boldsymbol{\psi}(\boldsymbol{\eta}-\tilde{\boldsymbol{q}})+\boldsymbol{\tau}_{e x t}, \\
\boldsymbol{B} \ddot{\boldsymbol{\eta}}+\boldsymbol{\psi}(\boldsymbol{\eta}-\tilde{\boldsymbol{q}}) & =\overline{\boldsymbol{u}} \\
\overline{\boldsymbol{u}} & =-\boldsymbol{K}_{P} \boldsymbol{\eta}-\boldsymbol{K}_{D} \dot{\boldsymbol{\eta}}
\end{aligned}
$$

where where $\tilde{\boldsymbol{q}} \equiv \boldsymbol{q}-\boldsymbol{q}_{d}(t)$ is the tracking error. $\boldsymbol{D} \in$ $\mathbb{R}^{n \times n}$ represents a symmetric, positive definite and bounded damping matrix. Note that $\boldsymbol{D}$ can be configuration dependent under the assumption that $\dot{\boldsymbol{D}}(\boldsymbol{q}, \boldsymbol{\eta})$ is bounded and that $\ddot{\boldsymbol{D}}(\boldsymbol{q}, \boldsymbol{\eta})$ is smooth, as will be required in sec. IV and sec. $\mathrm{V}$.

As will be shown later, the PD controller (22), with the symmetric, bounded and positive controller gain matrices $\boldsymbol{K}_{P}, \boldsymbol{K}_{D} \in \mathbb{R}^{n \times n}$, globally stabilizes the system and achieves tracking behavior $\tilde{\boldsymbol{q}}(t) \rightarrow \mathbf{0}, \boldsymbol{\eta}(t) \rightarrow \mathbf{0}$ as well as enforces link-side damping such that $\boldsymbol{q}(t)=$ const. $\Rightarrow \dot{\boldsymbol{q}} \rightarrow \mathbf{0}$ for the (partially) feedback linearized dynamics (20)-(21). The corresponding steady-state behavior is provided in V-B. Note, in contrast to the PD controller for the regulation case presented in II, $\boldsymbol{\eta}_{d}=\mathbf{0}^{4}$.

The link-side of the new, gravity-free system behaves like the original system (10) with additional damping and tracking. The motor dynamics in the new motor coordinates $\boldsymbol{\eta}$ are be structurally equal to original motor dynamics (11).

\footnotetext{
${ }^{3}$ The usual Euclidean norm for vectors and the corresponding induced matrix norm is assumed throughout the paper.

${ }^{4}$ The corresponding reference values $\boldsymbol{\eta}_{d}$ of controller (22) result, under the assumptions made in III, from the closed-loop link-side dynamics by imposing $\boldsymbol{q}(t)=\boldsymbol{q}_{d}(t)$.
} 


\section{B. Coordinate Transformation}

By comparing (10) and (20) we can find the relation

$$
\boldsymbol{\psi}(\boldsymbol{\theta}-\boldsymbol{q})-\boldsymbol{g}(\boldsymbol{q})=\boldsymbol{\psi}(\boldsymbol{\eta}-\tilde{\boldsymbol{q}})-\boldsymbol{D} \tilde{\boldsymbol{q}}-\boldsymbol{n}_{0}
$$

between the new coordinates $\boldsymbol{\eta}$ and the original system variables of (10)-(11), such that

$$
\boldsymbol{\eta}=\tilde{\boldsymbol{q}}+\boldsymbol{\psi}^{-1}\left(\boldsymbol{\psi}(\boldsymbol{\theta}-\boldsymbol{q})+\boldsymbol{D} \dot{\tilde{\boldsymbol{q}}}-\boldsymbol{g}(\boldsymbol{q})-\boldsymbol{n}_{0}\right),
$$

where ${ }^{5}$

$$
\boldsymbol{n}_{0}(\boldsymbol{q}, \dot{\boldsymbol{q}}, t) \equiv \boldsymbol{M}(\boldsymbol{q}) \ddot{\boldsymbol{q}}_{d}(t)+\boldsymbol{C}(\boldsymbol{q}, \dot{\boldsymbol{q}}) \ddot{\boldsymbol{q}}_{d}(t) .
$$

Since the inverse of $\psi$ is usually not analytically available (24) cannot be solved directly and $\boldsymbol{\eta}$ has to be determined numerically. For the experiments we use fix point iteration (see VIII).

Writing the motor dynamics in the new coordinates $\boldsymbol{\eta}$ requires the second time derivative of $\boldsymbol{\theta}$ as function of $\boldsymbol{\eta}$. To this end, we differentiate (23) w.r.t. time, i.e.

$$
\begin{array}{r}
\boldsymbol{\kappa}(\boldsymbol{\theta}-\boldsymbol{q})(\dot{\boldsymbol{\theta}}-\dot{\boldsymbol{q}})-\dot{\boldsymbol{g}}(\boldsymbol{q}) \\
=\boldsymbol{\kappa}(\boldsymbol{\eta}-\tilde{\boldsymbol{q}})(\dot{\boldsymbol{\eta}}-\dot{\tilde{\boldsymbol{q}}})-\frac{\mathrm{d}}{\mathrm{dt}}(\boldsymbol{D} \dot{\tilde{\boldsymbol{q}}})+\boldsymbol{n}_{1},
\end{array}
$$

where

$$
\begin{aligned}
& \boldsymbol{n}_{\mathbf{1}}(\boldsymbol{q}, \dot{\boldsymbol{q}}, \ddot{\boldsymbol{q}}, t) \equiv \frac{\mathrm{d}}{\mathrm{dt}}\left(\boldsymbol{n}_{\mathbf{0}}(\boldsymbol{q}, \dot{\boldsymbol{q}}, t)\right)=\dot{\boldsymbol{M}}(\boldsymbol{q}) \ddot{\boldsymbol{q}}_{d}(t) \\
& +\boldsymbol{M}(\boldsymbol{q}) \boldsymbol{q}_{d}^{(3)}(t)+\dot{\boldsymbol{C}}(\boldsymbol{q}, \dot{\boldsymbol{q}}) \dot{\boldsymbol{q}}_{d}(t)+\boldsymbol{C}(\boldsymbol{q}, \dot{\boldsymbol{q}}) \ddot{\boldsymbol{q}}_{d}(t)
\end{aligned}
$$

and

$$
\left.\boldsymbol{\kappa}\left(\phi_{0}\right) \equiv \frac{\partial^{2} U_{s}(\phi)}{\partial \phi_{i} \partial \phi_{j}}\right|_{\phi=\phi_{0}}=\left.\frac{\partial \boldsymbol{\psi}(\boldsymbol{\phi})}{\partial \boldsymbol{\phi}}\right|_{\phi=\phi_{0}} \in \mathbb{R}^{n \times n}
$$

is the local stiffness, i.e. the Hessian of the potential $U_{s}(\phi)$.

Using the second time derivative of (23)

$$
\begin{array}{r}
\dot{\boldsymbol{\kappa}}(\boldsymbol{\theta}-\boldsymbol{q})(\dot{\boldsymbol{\theta}}-\dot{\boldsymbol{q}})+\boldsymbol{\kappa}(\boldsymbol{\theta}-\boldsymbol{q})(\ddot{\boldsymbol{\theta}}-\ddot{\boldsymbol{q}})= \\
\dot{\boldsymbol{\kappa}}(\boldsymbol{\eta}-\tilde{\boldsymbol{q}})(\dot{\boldsymbol{\eta}}-\dot{\tilde{\boldsymbol{q}}})+\boldsymbol{\kappa}(\boldsymbol{\eta}-\tilde{\boldsymbol{q}})(\ddot{\boldsymbol{\eta}}-\ddot{\tilde{\boldsymbol{q}}}) \\
-\frac{\mathrm{d}^{2}}{\mathrm{dt}^{2}}(\boldsymbol{D} \dot{\tilde{\boldsymbol{q}}})+\ddot{\boldsymbol{g}}(\boldsymbol{q})+\boldsymbol{n}_{\mathbf{2}},
\end{array}
$$

where

$$
\dot{\boldsymbol{\kappa}}\left(\phi_{0}\right)=\frac{\mathrm{d}}{\mathrm{dt}} \boldsymbol{\kappa}(\phi(t))=\left.\left(\sum_{k=1}^{n} \frac{\partial \boldsymbol{\kappa}}{\partial \phi_{k}} \dot{\phi}_{k}\right)\right|_{\phi=\phi_{0}} \in \mathbb{R}^{n \times n}
$$

and

$$
\begin{aligned}
& \boldsymbol{n}_{\mathbf{2}}\left(\boldsymbol{q}, \dot{\boldsymbol{q}}, \ddot{\boldsymbol{q}}, \boldsymbol{q}^{(3)}, t\right) \equiv \frac{\mathrm{d}^{2}}{\mathrm{dt}^{2}} \boldsymbol{n}_{\mathbf{0}}=\ddot{\boldsymbol{M}}(\boldsymbol{q}) \ddot{\boldsymbol{q}}_{d}+2 \dot{\boldsymbol{M}}(\boldsymbol{q}) \boldsymbol{q}_{d}^{(3)} \\
+ & \boldsymbol{M}(\boldsymbol{q}) \boldsymbol{q}_{d}^{(4)}+\ddot{\boldsymbol{C}}(\boldsymbol{q}, \dot{\boldsymbol{q}}) \dot{\boldsymbol{q}}_{d}+2 \dot{\boldsymbol{C}}(\boldsymbol{q}, \dot{\boldsymbol{q}}) \ddot{\boldsymbol{q}}_{d}+\boldsymbol{C}(\boldsymbol{q}, \dot{\boldsymbol{q}}) \boldsymbol{q}_{d}^{(3)}
\end{aligned}
$$

\footnotetext{
${ }^{5}$ With better readability in mind the arguments of $\boldsymbol{n}_{0}$ and its time derivatives $\left(\boldsymbol{n}_{1}, \boldsymbol{n}_{2}\right.$; see below) will be left out in the remainder of this text, once they have been introduced.
}

allows us to express $\ddot{\theta}$ as function of $\boldsymbol{\eta}, \dot{\boldsymbol{\eta}}$ and $\ddot{\eta}$

$$
\begin{aligned}
\ddot{\boldsymbol{\theta}}= & \ddot{\boldsymbol{q}}+\boldsymbol{\kappa}^{-1}(\boldsymbol{\theta}-\boldsymbol{q})(-\dot{\boldsymbol{\kappa}}(\boldsymbol{\theta}-\boldsymbol{q})(\dot{\boldsymbol{\theta}}-\dot{\boldsymbol{q}}) \\
& +\dot{\boldsymbol{\kappa}}(\boldsymbol{\eta}-\tilde{\boldsymbol{q}})(\dot{\boldsymbol{\eta}}-\dot{\tilde{\boldsymbol{q}}})+\boldsymbol{\kappa}(\boldsymbol{\eta}-\tilde{\boldsymbol{q}})(\ddot{\boldsymbol{\eta}}-\ddot{\tilde{\boldsymbol{q}}}) \\
& \left.-\frac{\mathrm{d}^{2}}{\mathrm{dt}^{2}}(\boldsymbol{D} \dot{\tilde{\boldsymbol{q}}})+\ddot{\boldsymbol{g}}(\boldsymbol{q})+\boldsymbol{n}_{\mathbf{2}}\right) .
\end{aligned}
$$

Relations for $\dot{\eta}$ and $\ddot{\eta}$ can be found in VIII.

Relations for $\dot{\boldsymbol{\eta}}$ and $\ddot{\boldsymbol{\eta}}$ as function of $\boldsymbol{\theta}, \dot{\boldsymbol{\theta}}$ and $\boldsymbol{\theta}, \dot{\boldsymbol{\theta}}, \ddot{\boldsymbol{\theta}}$, respectively, can be derived in analog fashion.

\section{Partial Feedback Linearization of the Motor Dynamics}

Substituting $\boldsymbol{\psi}(\boldsymbol{\theta}-\boldsymbol{q})$ and $\ddot{\boldsymbol{\theta}}$ from (23) and (32) into (11) yields the transformed motor dynamics

$$
\begin{aligned}
& \boldsymbol{B} \ddot{\boldsymbol{q}}+\boldsymbol{B}\left(\boldsymbol{\kappa}^{-1}(\boldsymbol{\theta}-\boldsymbol{q})(-\dot{\boldsymbol{\kappa}}(\boldsymbol{\theta}-\boldsymbol{q})(\dot{\boldsymbol{\theta}}-\dot{\boldsymbol{q}})\right. \\
& +\dot{\boldsymbol{\kappa}}(\boldsymbol{\eta}-\tilde{\boldsymbol{q}})(\dot{\boldsymbol{\eta}}-\dot{\tilde{\boldsymbol{q}}})+\boldsymbol{\kappa}(\boldsymbol{\eta}-\tilde{\boldsymbol{q}})(\ddot{\boldsymbol{\eta}}-\ddot{\tilde{\boldsymbol{q}}})-\frac{\mathrm{d}^{2}}{\mathrm{dt}^{2}}(\boldsymbol{D} \dot{\tilde{\boldsymbol{q}}}) \\
& \left.\left.+\ddot{\boldsymbol{g}}(\boldsymbol{q})+\boldsymbol{n}_{\mathbf{2}}\right)\right)+\boldsymbol{\psi}(\boldsymbol{\eta}-\tilde{\boldsymbol{q}})-\boldsymbol{D} \dot{\tilde{\boldsymbol{q}}}+\boldsymbol{g}(\boldsymbol{q})+\boldsymbol{n}_{\mathbf{0}}=\boldsymbol{\tau}_{m} .
\end{aligned}
$$

To achieve closed-loop motor dynamics that structurally resemble the desired motor dynamics (21) we perform a controller design in two steps.

First, we pre-compensate some undesired nonlinear terms via $\boldsymbol{\tau}_{m}=\hat{\boldsymbol{u}}+\check{\boldsymbol{u}}$, where

$$
\begin{aligned}
\hat{\boldsymbol{u}}= & \boldsymbol{B} \ddot{\boldsymbol{q}}+\boldsymbol{B}\left(\boldsymbol{\kappa}^{-1}(\boldsymbol{\theta}-\boldsymbol{q})(-\dot{\boldsymbol{\kappa}}(\boldsymbol{\theta}-\boldsymbol{q})(\dot{\boldsymbol{\theta}}-\dot{\boldsymbol{q}})\right. \\
& +\dot{\boldsymbol{\kappa}}(\boldsymbol{\eta}-\tilde{\boldsymbol{q}})(\dot{\boldsymbol{\eta}}-\dot{\tilde{\boldsymbol{q}}})-\boldsymbol{\kappa}(\boldsymbol{\eta}-\tilde{\boldsymbol{q}}) \ddot{\tilde{\boldsymbol{q}}}-\frac{\mathrm{d}^{2}}{\mathrm{dt}^{2}}(\boldsymbol{D} \dot{\tilde{\boldsymbol{q}}}) \\
& \left.\left.+\ddot{\boldsymbol{g}}(\boldsymbol{q})+\boldsymbol{n}_{\mathbf{2}}\right)\right)-\boldsymbol{D} \dot{\tilde{\boldsymbol{q}}}+\boldsymbol{g}(\boldsymbol{q})+\boldsymbol{n}_{\mathbf{0}},
\end{aligned}
$$

resulting in the following motor dynamics

$$
\boldsymbol{B} \boldsymbol{\kappa}^{-1}(\boldsymbol{\theta}-\boldsymbol{q}) \boldsymbol{\kappa}(\boldsymbol{\eta}-\tilde{\boldsymbol{q}}) \ddot{\boldsymbol{\eta}}+\boldsymbol{\psi}(\boldsymbol{\eta}-\tilde{\boldsymbol{q}})=\check{\boldsymbol{u}} .
$$

with the intermediate control input $\check{\boldsymbol{u}}$. Note, that in case of a state-dependent damping matrix an implicit equation for $\boldsymbol{u}$ results which always can be solved.

The difference to the desired motor dynamics (21) is the state-dependent inertia matrix $\boldsymbol{B} \boldsymbol{\kappa}^{-1}(\boldsymbol{\theta}-\boldsymbol{q}) \boldsymbol{\kappa}(\boldsymbol{\eta}-\tilde{\boldsymbol{q}})$, compared to the constant inertia $\boldsymbol{B}$. To achieve constant inertia $\boldsymbol{B}$ in (39), the intermediary control input $\check{\boldsymbol{u}}$ can be chosen as

$$
\begin{aligned}
\check{\boldsymbol{u}}= & \left(\boldsymbol{I}-\boldsymbol{B} \boldsymbol{\kappa}^{-1}(\boldsymbol{\theta}-\boldsymbol{q}) \boldsymbol{\kappa}(\boldsymbol{\eta}-\tilde{\boldsymbol{q}}) \boldsymbol{B}^{-1}\right) \boldsymbol{\psi}(\boldsymbol{\eta}-\tilde{\boldsymbol{q}}) \\
& +\boldsymbol{B} \boldsymbol{\kappa}^{-1}(\boldsymbol{\theta}-\boldsymbol{q}) \boldsymbol{\kappa}(\boldsymbol{\eta}-\tilde{\boldsymbol{q}}) \boldsymbol{B}^{-1} \overline{\boldsymbol{u}}
\end{aligned}
$$

which yields the desired motor dynamics in (21).

The final controller input $\tau_{m}$ is composed of eqs. (22), (34) and (36)

$$
\boldsymbol{\tau}_{m}=\hat{\boldsymbol{u}}+\check{\boldsymbol{u}}(\overline{\boldsymbol{u}}),
$$

where $\check{\boldsymbol{u}}$ is a function of $\overline{\boldsymbol{u}}$. 


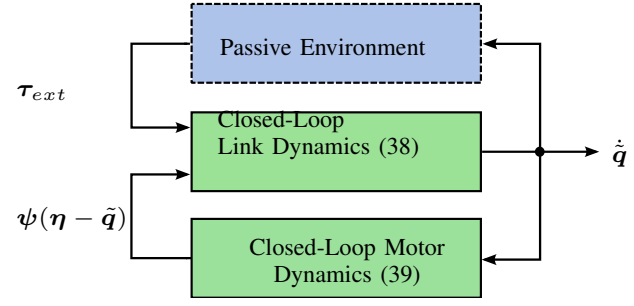

Fig. 3. Representation of the closed-loop dynamics (38)-(39) as an interconnection of passive subsystems and its respective power ports.

\section{Stability \& Passivity Analysis}

This section analyses the passivity properties and derives a stability statement of the closed-loop dynamics

$$
\begin{array}{r}
\boldsymbol{M}(\tilde{\boldsymbol{q}}, t) \ddot{\tilde{\boldsymbol{q}}}+(\boldsymbol{C}(\tilde{\boldsymbol{q}}, \dot{\tilde{\boldsymbol{q}}}, t)+\boldsymbol{D}(\tilde{\boldsymbol{q}}, \boldsymbol{\eta}, t)) \dot{\tilde{\boldsymbol{q}}}=\boldsymbol{\psi}(\boldsymbol{\eta}-\tilde{\boldsymbol{q}}, t)+\boldsymbol{\tau}_{e x t}, \\
\boldsymbol{B} \ddot{\boldsymbol{\eta}}+\boldsymbol{\psi}(\boldsymbol{\eta}-\tilde{\boldsymbol{q}}, t)=-\boldsymbol{K}_{P} \boldsymbol{\eta}-\boldsymbol{K}_{D} \dot{\boldsymbol{\eta}}
\end{array}
$$

resulting for the plant dynamics (10)-(11) together with the controller (37), written in tracking error variables, the timevariant behavior becomes immediately apparent.

\section{A. Passivity}

On the basis of definition [24] it will be shown that the link dynamics (38) and the closed-loop motor dynamics (39) can be presented as an interconnection of passive subsystems [24] as depicted in Fig. 3. For the link side dynamics we consider the time varying storage function

$$
S_{\tilde{q}}=\frac{1}{2} \dot{\tilde{\boldsymbol{q}}}^{\mathrm{T}} \boldsymbol{M}(\tilde{\boldsymbol{q}}, t) \dot{\tilde{\boldsymbol{q}}}
$$

For the analysis of the controller proposed in this work, we express the derivative of (40) in the form,

$$
\dot{S}_{\tilde{q}}=-\dot{\tilde{\boldsymbol{q}}}^{\mathrm{T}} \boldsymbol{D} \dot{\tilde{\boldsymbol{q}}}+\dot{\tilde{\boldsymbol{q}}}^{\mathrm{T}} \boldsymbol{\psi}(\boldsymbol{\eta}-\tilde{\boldsymbol{q}})+\dot{\tilde{\boldsymbol{q}}}^{\mathrm{T}} \boldsymbol{\tau}_{\mathrm{ext}},
$$

which corresponds to the representation of the closed-loop link dynamics given in $(38)^{6}$. On the right hand side of (41) one can identify two terms, each corresponding to an interconnection port with the link dynamics. The second term represents the interconnection with the controlled motor dynamics (39) and the third term represents the interconnection with an external system. For the former we consider the storage function of the form

$$
S_{\eta}=\frac{1}{2} \dot{\boldsymbol{\eta}}^{\mathrm{T}} \boldsymbol{B} \dot{\boldsymbol{\eta}}+U_{\boldsymbol{\tau}}(\boldsymbol{\eta}-\tilde{\boldsymbol{q}})+\frac{1}{2} \boldsymbol{\eta}^{\mathrm{T}} \boldsymbol{K}_{P} \boldsymbol{\eta} .
$$

The first term represents a virtual kinetic energy of the motorside, while the second term represents the total potential energy stored in virtual springs and the third term represents a virtual control energy. Its derivative along the solution of the closed-loop motor dynamics (39) can be expressed as

$$
\dot{S}_{\eta}=-\dot{\boldsymbol{\eta}}^{\mathrm{T}} \boldsymbol{K}_{D} \dot{\boldsymbol{\eta}}-\dot{\tilde{\boldsymbol{q}}}^{\mathrm{T}} \boldsymbol{\psi}(\boldsymbol{\eta}-\tilde{\boldsymbol{q}})
$$

${ }^{6}$ Note that (10) and (38) are equivalent.
Comparing (41) and (43), the interconnection between the link and motor dynamics becomes directly apparent. The above analysis motivates the following proposition.

Proposition 1: The system (38)-(39) can be represented as a passive map from the generalized external forces $\tau_{\text {ext }}$ to the generalized velocity of the link-side tracking error $\dot{\tilde{q}}$.

Proof: Consider the storage function $S=S_{\tilde{q}}+S_{\eta}$ comprising (40) and (42) of which the derivative along the solution of the system (38)-(39) satisfies

$$
\dot{S}=-\dot{\tilde{\boldsymbol{q}}}^{\mathrm{T}} \boldsymbol{D} \dot{\tilde{\boldsymbol{q}}}-\dot{\boldsymbol{\eta}}^{\mathrm{T}} \boldsymbol{K}_{D} \dot{\boldsymbol{\eta}}+\dot{\tilde{\boldsymbol{q}}}^{\mathrm{T}} \boldsymbol{\tau}_{\mathrm{ext}} \leq \dot{\tilde{\boldsymbol{q}}} \tau_{\mathrm{ext}},
$$

which completes the proof according to [24].

Note, for the special case of regulation control, i.e. $\boldsymbol{q}_{d}=$ const., the closed-loop system can be seen as a passive mapping from the environment with respect to the, physically more intuitive, power port $\dot{\boldsymbol{q}}^{\mathrm{T}}, \boldsymbol{\tau}_{\text {ext }}$.

\section{B. Stability, Steady-State Behavior}

First, we determine the steady state conditions for the closed loop dynamics. Second, we deduce stability by considering the storage function of proof of Proposition 1 as Lyapunov function candidate.

By setting the state derivatives $(\dot{\tilde{\boldsymbol{q}}}, \dot{\boldsymbol{\eta}})$ to zero, the steady state conditions for (38)-(39), in absence of external loads, become

$$
\begin{aligned}
\dot{\tilde{\boldsymbol{q}}}_{0} & =\mathbf{0}, \\
\dot{\boldsymbol{\eta}}_{0} & =\mathbf{0}, \\
\boldsymbol{\psi}\left(\boldsymbol{\eta}_{0}-\tilde{\boldsymbol{q}}_{0}\right) & =\mathbf{0}, \\
\boldsymbol{\psi}\left(\boldsymbol{\eta}_{0}-\tilde{\boldsymbol{q}}_{0}\right)-\boldsymbol{K}_{P} \boldsymbol{\eta}_{0} & =\mathbf{0} .
\end{aligned}
$$

It can be easily verified that (45)-(46) have a unique solution

$$
\begin{aligned}
\tilde{\boldsymbol{q}}_{0} & =\mathbf{0} \\
\boldsymbol{\eta}_{0} & =\mathbf{0}
\end{aligned}
$$

under assumptions in III and if $\boldsymbol{K}_{P}$ is positive definite.

Proposition 2: Under the assumptions made in III, the closed-loop dynamics (38)-(39) is uniformly stable.

Proof: We show stability by invoking Lyapunov's direct method for non-autonomous systems [25]. For that we have to find a scalar, positive definite function $V(\boldsymbol{x}, t)$ with continuous partial derivatives such that $\dot{V}$ is negative semidefinite. Furthermore, for uniform stability, $V(\boldsymbol{x}, t)$ has to be upper bounded by a time-invariant positive definite function $V^{*}(\boldsymbol{x})$ such that $V(\boldsymbol{x}, t) \leq V^{*}(\boldsymbol{x}) \forall t$ [25]. Consider

$$
V(\boldsymbol{x}, t)=S_{\tilde{q}}(\tilde{\boldsymbol{q}}, \dot{\tilde{\boldsymbol{q}}}, t)+S_{\eta}(\boldsymbol{\eta}, \dot{\boldsymbol{\eta}}, \tilde{\boldsymbol{q}}, t)
$$

as an energy based, time-variant Lyapunov function, where $\boldsymbol{x}=\left(\tilde{\boldsymbol{q}}^{\mathrm{T}}, \boldsymbol{\eta}^{\mathrm{T}}, \dot{\tilde{\boldsymbol{q}}}^{\mathrm{T}}, \dot{\boldsymbol{\eta}}^{\mathrm{T}}\right)^{\mathrm{T}}$. Obviously, $V(\boldsymbol{x}, t)$ is positive definite since $\boldsymbol{M}, \boldsymbol{B}$ and $\boldsymbol{K}_{P}$ are positive definite and the spring potential is, by assumption, positive semi-definite. Let us choose $V^{*}$ as follows

$$
V^{*}(\boldsymbol{x})=\overline{\boldsymbol{M}}\|\dot{\tilde{\boldsymbol{q}}}\|^{2}+\dot{\boldsymbol{\eta}}^{\mathrm{T}} \boldsymbol{B} \dot{\boldsymbol{\eta}}+U_{\boldsymbol{\tau}}(\boldsymbol{\eta}-\tilde{\boldsymbol{q}})+\boldsymbol{\eta}^{\mathrm{T}} \boldsymbol{K}_{P} \boldsymbol{\eta},
$$

where $\overline{\boldsymbol{M}} \equiv \max \lambda(\boldsymbol{M}(\tilde{\boldsymbol{q}}, t))$. Note, $\overline{\boldsymbol{M}}$ exists and is bounded as the inertia matrix $\boldsymbol{M}(\boldsymbol{q})$ is bounded [26]. The 
time derivative of $V$ along the solutions of the closed loop system (38)-(39) is given by ${ }^{7}$

$$
\dot{V}(\tilde{\boldsymbol{q}}, \dot{\boldsymbol{q}}, \boldsymbol{\eta}, \dot{\boldsymbol{\eta}}, t)=-\dot{\tilde{\boldsymbol{q}}}^{\mathrm{T}} \boldsymbol{D} \dot{\tilde{\boldsymbol{q}}}-\dot{\boldsymbol{\eta}}^{\mathrm{T}} \boldsymbol{K}_{D} \dot{\boldsymbol{\eta}}
$$

Since $\boldsymbol{D}$ and $\boldsymbol{K}_{D}$ are positive definite, $\dot{V}$ is negative semidefinite. This completes the proof.

By invoking Barbalat's lemma [25]

Lemma 1: If a scalar function $V(\boldsymbol{x}, t)$ satisfies the following conditions

- $V(\boldsymbol{x}, t)$ is lower bounded

- $\dot{V}(\boldsymbol{x}, t)$ is negative semi-definite

- $\dot{V}(\boldsymbol{x}, t)$ is uniformly continuous in time

then $\dot{V}(\boldsymbol{x}, t) \rightarrow 0$ as $t \rightarrow \infty$.

we can show asymptotic convergence of the generalized velocities of the tracking error, i.e. $\dot{\boldsymbol{q}}(t) \rightarrow \dot{\boldsymbol{q}}_{d}(t)$ and $\dot{\boldsymbol{\eta}}(t) \rightarrow \mathbf{0}$, for the closed-loop dynamics (38)-(39).

Proof: To complete the proof only condition 3 is left to show. The second time derivative of $V$ is given by

$$
\begin{aligned}
\ddot{V}(\boldsymbol{x}, t)= & 2\left(\dot{\tilde{\boldsymbol{q}}}^{\mathrm{T}} \boldsymbol{D} \boldsymbol{M}^{-1} \boldsymbol{C} \dot{\tilde{\boldsymbol{q}}}+\dot{\tilde{\boldsymbol{q}}}^{\mathrm{T}} \boldsymbol{D} \boldsymbol{M}^{-1} \boldsymbol{D} \dot{\tilde{\boldsymbol{q}}}\right. \\
& \left.-\dot{\tilde{\boldsymbol{q}}}^{\mathrm{T}} \boldsymbol{D} \boldsymbol{M}^{-1} \boldsymbol{\psi}(\boldsymbol{\eta}-\tilde{\boldsymbol{q}})\right)-\dot{\tilde{\boldsymbol{q}}}^{\mathrm{T}} \dot{\boldsymbol{D}} \tilde{\boldsymbol{q}} \\
& +2\left(\dot{\boldsymbol{\eta}}^{\mathrm{T}} \boldsymbol{K}_{D} \boldsymbol{B}^{-1} \boldsymbol{\psi}(\boldsymbol{\eta}-\tilde{\boldsymbol{q}})\right. \\
& \left.+\dot{\boldsymbol{\eta}}^{\mathrm{T}} \boldsymbol{K}_{D} \boldsymbol{B}^{-1} \boldsymbol{K}_{P} \boldsymbol{\eta}+\dot{\boldsymbol{\eta}}^{\mathrm{T}} \boldsymbol{K}_{D} \boldsymbol{B}^{-1} \boldsymbol{K}_{D} \dot{\boldsymbol{\eta}}\right)
\end{aligned}
$$

From the stability prove above, we already know that $\tilde{\boldsymbol{q}}, \boldsymbol{\eta}, \dot{\tilde{\boldsymbol{q}}}$ and $\dot{\eta}$ are bounded. $M, M^{-1}, C, B$ are obviously bounded under the assumptions made in III. $\boldsymbol{D}$ and $\dot{D}$ are assumed to be bounded (see III). The sequence of bounded matrices is again bounded. Thereby we can deduce that $\ddot{V}$ is bounded, which again, is a sufficient condition for $\dot{V}$ to be uniformly continuous [25]. Barbalat's lemma then states that $\dot{V} \rightarrow 0$, which, due the positive definite nature of $\boldsymbol{M}$ and $\boldsymbol{K}_{D}$, can only be the case if $\dot{\boldsymbol{\eta}}, \dot{\tilde{\boldsymbol{q}}} \rightarrow \mathbf{0}$.

\section{ExPerimental VAlidation And PeRformance ANALYSIS}

The performance of the control approach has been experimentally evaluated on the first four Variable Stiffness Actuators (VSA) joints of the DLR Hand Arm System [1], see also fig. 1. The first four arm joints, namely the elbow and the three shoulder joints, are implemented by Floating Spring Joints (FSJ) [27].

First, we present one possible layout of the damping matrix $\boldsymbol{D}$ based on modal decomposition. Note, the control approach presented in this paper doesn't restrict the user to a specific damping matrix layout. The only restrictions on $D$ are the assumptions made in IV-A. In the latter part we present the experimental results.

\footnotetext{
${ }^{7}$ The symmetry of $\boldsymbol{K}_{P}$ is exploited while transforming the equations.
}

\section{A. Damping Design}

The entries of the inertia matrix of robotic systems with rotational joints vary strongly with the kinematic configuration of the robot. Additionally, the (local) stiffness of many variable stiffness mechanisms varies with varying load. To achieve similar performance in the complete workspace of the robot, an approach which accounts for these variations should be considered. A damping design which accounts for the variation of the inertia and stiffness matrix can be derived from the generalized eigenvalue problem of the linearized system

$$
\boldsymbol{M}(\boldsymbol{q}) \ddot{\boldsymbol{q}}+\boldsymbol{D}(\boldsymbol{q}, \boldsymbol{\eta}) \dot{\boldsymbol{q}}-\boldsymbol{\kappa}(\boldsymbol{\eta}-\boldsymbol{q})(\boldsymbol{\eta}-\boldsymbol{q})=\mathbf{0}
$$

such that

$$
\boldsymbol{D}(\boldsymbol{q}, \boldsymbol{\eta})=2 \boldsymbol{Q}(\boldsymbol{q}, \boldsymbol{\eta})^{-T} \operatorname{diag}\left(\xi_{i} \sqrt{\lambda_{i}}\right) \boldsymbol{Q}(\boldsymbol{q}, \boldsymbol{\eta})^{-1} .
$$

Herein, $Q \in \mathbb{R}^{n \times n}$ is a non-singular matrix satisfying $\boldsymbol{Q}^{T} \boldsymbol{Q}=\boldsymbol{M}$ and $\boldsymbol{\kappa}=\boldsymbol{Q}^{T} \boldsymbol{\Lambda} \boldsymbol{Q}$, where $\boldsymbol{\Lambda}$ is a diagonal matrix containing the real and positive eigenvalues $\lambda_{i}$, since $\boldsymbol{M}$ is a symmetric and positive definite matrix and $\kappa$ is a symmetric matrix ([28]). Further, $\xi_{i}>0$ are normalized damping factors sometimes referred to modal damping. Note that the instantaneous linearization is only performed for the design of the damping matrix.

Remark 2: This damping design implies the matrix $\boldsymbol{D}(\boldsymbol{q}, \boldsymbol{\eta})$ to be a function of the link variables $\boldsymbol{q}$ and motor variables $\boldsymbol{\eta}$. Substitution of $\frac{\mathrm{d}^{2}}{\mathrm{~d} t^{2}}(\boldsymbol{D}(\boldsymbol{q}, \boldsymbol{\eta}) \dot{\tilde{\boldsymbol{q}}})$ in (34) would result, as previously mentioned, in an algebraic loop which can always be solved. In addition, the first and second time derivatives of eigenvalues and eigenvectors of (56) have to be calculated. The interested read can find more details about this issue in [29] and [30].

Remark 3: Simulations have shown, that for our case, neglecting terms, containing $\dot{D}$ and $\ddot{D}$, does not significantly impact the controller performance. Therefore these terms are dropped for the experiments.

\section{B. Results}

Three different experiments have been performed to analyze the controller performance. Throughout all experiments the damping design presented in VI-A has been used to layout $\boldsymbol{D}$, with $\xi_{q_{i}}=0.5, i=1, \ldots, 4 . \boldsymbol{K}_{P}$ was manually tuned such the that the control input $\boldsymbol{\tau}_{m}$ doesn't overshoot its limits and was set to $\boldsymbol{K}_{P}=\operatorname{diag}\left(\left[\begin{array}{ll}1000 & 900750750\end{array}\right]\right)$ $\mathrm{Nm} . \boldsymbol{K}_{D}$ is set such that the (decoupled) motor dynamics are critically damped $\left(K_{D}\right)_{i j=1 \ldots 4}=2 \delta_{i j} \xi_{x_{i}} \sqrt{K_{P_{i j}} B_{i j}}, \xi_{x_{i}}=$ $0.7 \forall i$. The stiffness adjustment was set to $\sigma_{i}=5 \mathrm{deg} \forall i$; corresponding to the mid curve of the stiffness plot in fig. 1.

First, we show the tracking performance in absence of external disturbances, see fig. 4 . In this experiment the maximum angular velocities are $\dot{\boldsymbol{q}}^{\mathrm{T}}=\left[\begin{array}{llll}190 & 30 & 103 & 113\end{array}\right] \mathrm{deg} / \mathrm{s}$. Note, between each of three motions, at time points $t_{1}$ and $t_{2}$, $\boldsymbol{q}_{d}$ is adjusted to the current values of $\boldsymbol{q}$. Model and sensor uncertainties are sources for the non-zero steady state error.

In the second experiment, we show the damping performance. Figure 5.a shows the response of a classical motor PD controller. Figure 5.b shows of our proposed controller. 

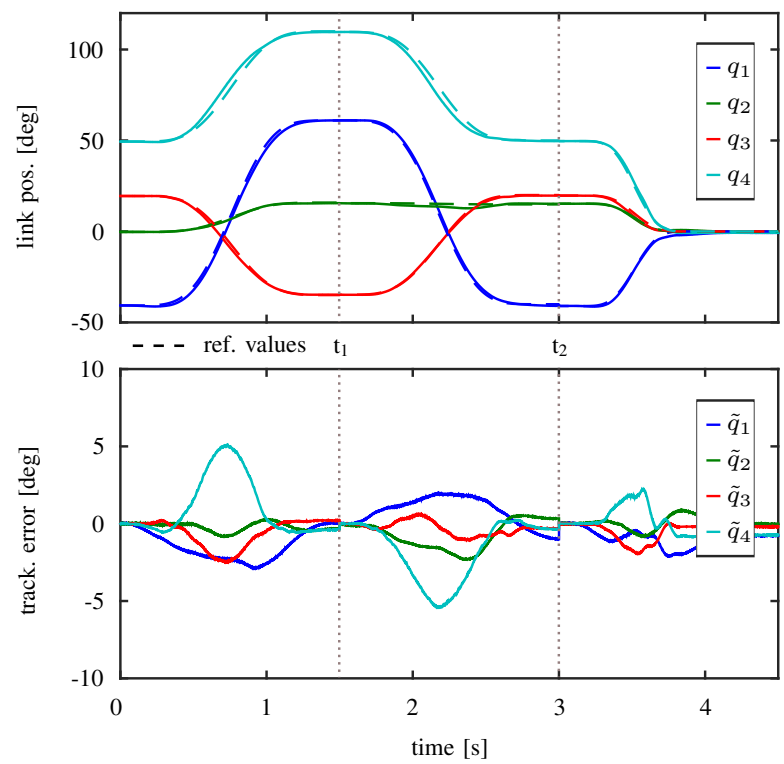

Fig. 4. Experimental result: tracking performance in absence of external disturbances. At time points $t_{1}$ and $t_{2}, \boldsymbol{q}_{d}$ is adjusted to the current values of $\boldsymbol{q}$. The maximum angular velocities reached reached $\dot{\boldsymbol{q}}^{\mathrm{T}}=$ [190 30103 113] deg/s.

In a stationary configuration of $\boldsymbol{q}=\left[\begin{array}{llll}11061 & 16-35\end{array}\right]^{\mathrm{T}} \mathrm{deg}$ an external torque was applied to system through physical human interaction. The magnitude of disturbance can be best seen on peaks in the respective joint torques $\max _{t}\left(\boldsymbol{\tau}_{j}\right)=$ $\left[\begin{array}{llll}68 & 63 & 17 & -28\end{array}\right]^{\mathrm{T}} \mathrm{Nm}$. The deflections in joint one and two are close to the maximum the system can handle.

It can be seen that the convergence rate of the link variables in case of our controller is about 8 times higher compared to the classical PD controller.

Figure 6 show the disturbance rejection performance while moving on a trajectory. Again the system is disturbed through physical human interaction. Within $0.3 \mathrm{~s}$ the system converges into an error band of \pm 1 deg.

A Video showing all three experiments can be found as attachment.

\section{CONLUSION}

This paper proposes a control law to implement an exact link-side trajectory tracking and disturbance rejection behavior while changing the original plant dynamics only to a minimum extend. This is achieved by introducing new coordinates for the motor reflecting these damping and feedforward terms. The motor dynamics in the new coordinates structurally equal the original motor dynamics such that PD regulation control achieves the desired linkside behavior. This in turn allows for a comprehensive stability and passivity analysis based on physically motivated Lyapunov functions. The performance of the approach has been experimentally evaluated on a multi-joint VSA robot arm. As for any link-side tracking controller, a theoretical limitation of the approach seemed to be the usage of the second and third time derivative of the measured link position in the control law, which however turned out not to be a practical limitation, since the time derivatives could be computed based on an accurate model of the plant. To our best knowledge, this is the first link-side tracking controller for compliantly actuated robots with nonlinear elastic transmissions, which is theoretically founded and reported to be experimentally validated on a multi degrees of freedom robot.

\section{APPENDIX \\ REFERENCES}

[1] A. Albu-Schäffer, O. Eiberger, M. Fuchs, M. Grebenstein, S. Haddadin, C. Ott, A. Stemmer, T. Wimbck, S. Wolf, C. Borst, and G. Hirzinger, "Anthropomorphic soft robotics from torque control to variable intrinsic compliance," in Robotics Research, ser. Springer Tracts in Advanced Robotics, C. Pradalier, R. Siegwart, and G. Hirzinger, Eds. Springer, 2011, vol. 70, pp. 185-207.

[2] D. Lakatos and A. Albu-Schäffer, "Switching based limit cycle control for compliantly actuated second-order systems," in Proceedings of the 19th IFAC World Congress, 2014, pp. 6392-6399.

[3] P. Tomei, "A simple pd controller for robots with elastic joints," Automatic Control, IEEE Transactions on, vol. 36, no. 10, pp. 12081213 , Oct 1991.

[4] R. Ortega, R. Kelly, and A. Loria, "A class of output feedback globally stabilizing controllers for flexible joints robots," Robotics and Automation, IEEE Transactions on, vol. 11, no. 5, pp. 766-770, Oct 1995.

[5] L. Zollo, A. De Luca, and B. Siciliano, "Regulation with on-line gravity compensation for robots with elastic joints," in Robotics and Automation, 2004. Proceedings. ICRA '04. 2004 IEEE International Conference on, vol. 3, April 2004, pp. 2687-2692.

[6] C. Ott, A. Albu-Schäffer, A. Kugi, S. Stamigioli, and G. Hirzinger, "A passivity based cartesian impedance controller for flexible joint robots-part i: Torque feedback and gravity compensation," in Proc. IEEE Int. Conf. on Robotics and Automation, 2004.

[7] B. Vanderborght, A. Albu-Schäffer, A. Bicchi, E. Burdet, D. G. Caldwell, R. Carloni, M. Catalano, O. Eiberger, W. Friedl, G. Ganesh et al., "Variable impedance actuators: A review," Robotics and Autonomous Systems, vol. 61, no. 12, pp. 1601-1614, 2013.

[8] A. Albu-Schäffer, C. Ott, and F. Petit, "Constructive energy shaping control for a class of euler-lagrange systems," in 10th Int. IFAC Symposium on Robot Control, 2012.

[9] A. Albu-Schäffer and G. Hirzinger, "A globally stable state feedback controller for flexible joint robots," Advanced Robotics, vol. 15, no. 8, pp. 799-814, 2001.

[10] F. Petit and A. Albu-Schäffer, "State feedback damping control for a multi dof variable stiffness robot arm," in Proc. IEEE Int. Conf. on Robotic and Automation, 2011.

[11] I. Sardellitti, G. Medrano-Cerda, N. Tsagarakis, A. Jafari, and D. Caldwell, "Gain scheduling control for a class of variable stiffness actuators based on lever mechanisms," Robotics, IEEE Transactions on, vol. 29, no. 3, pp. 791-798, June 2013.

[12] M. W. Spong, "Modeling and control of elastic joint robots," Transactions of the ASME: Journal of Dynamic Systems, Measurement, and Control, vol. 109, pp. 310-319, 1987.

[13] S. Nicosia and P. Tomei, "Design of global tracking controllers for flexible-joint robots," Journal of robotic systems, vol. 10, no. 6, pp. 835-846, 1993.

[14] A. Loria and R. Ortega, "On tracking control of rigid and flexible joints robots," Appl. Math. Comput. Sci, vol. 5, no. 2, pp. 101-113, 1995.

[15] C. Ott, A. Albu-Schäffer, A. Kugi, and G. Hirzinger, "Decoupling based cartesian impedance control of flexible joint robots," in Proc. IEEE Int. Conf. on Robotics and Automation, 2003.

[16] J. H. Oh and J. S. Lee, "Control of flexible joint robot system by backstepping design approach," Intelligent Automation \& Soft Computing, vol. 5, no. 4, pp. 267-278, 1999.

[17] C. Ott, Cartesian Impedance Control of Redundant and Flexible-Joint Robots, B. Siciliano and O. Khatib, Eds. Springer, 2008.

[18] J.-J. Slotine and L. Weiping, "Adaptive manipulator control: A case study," Automatic Control, IEEE Transactions on, vol. 33, no. 11, pp. 995-1003, 1988.

[19] M. W. Spong, "Adaptive control of flexible joint manipulators," Systems \& Control Letters, vol. 13, no. 1, pp. 15-21, 1989.

[20] A. De Luca and P. Lucibello, "A general algorithm for dynamic feedback linearization of robots with elastic joints," in IEEE Int. Conf. on Robotics and Automation, vol. 1, 1998, pp. 504-510. 

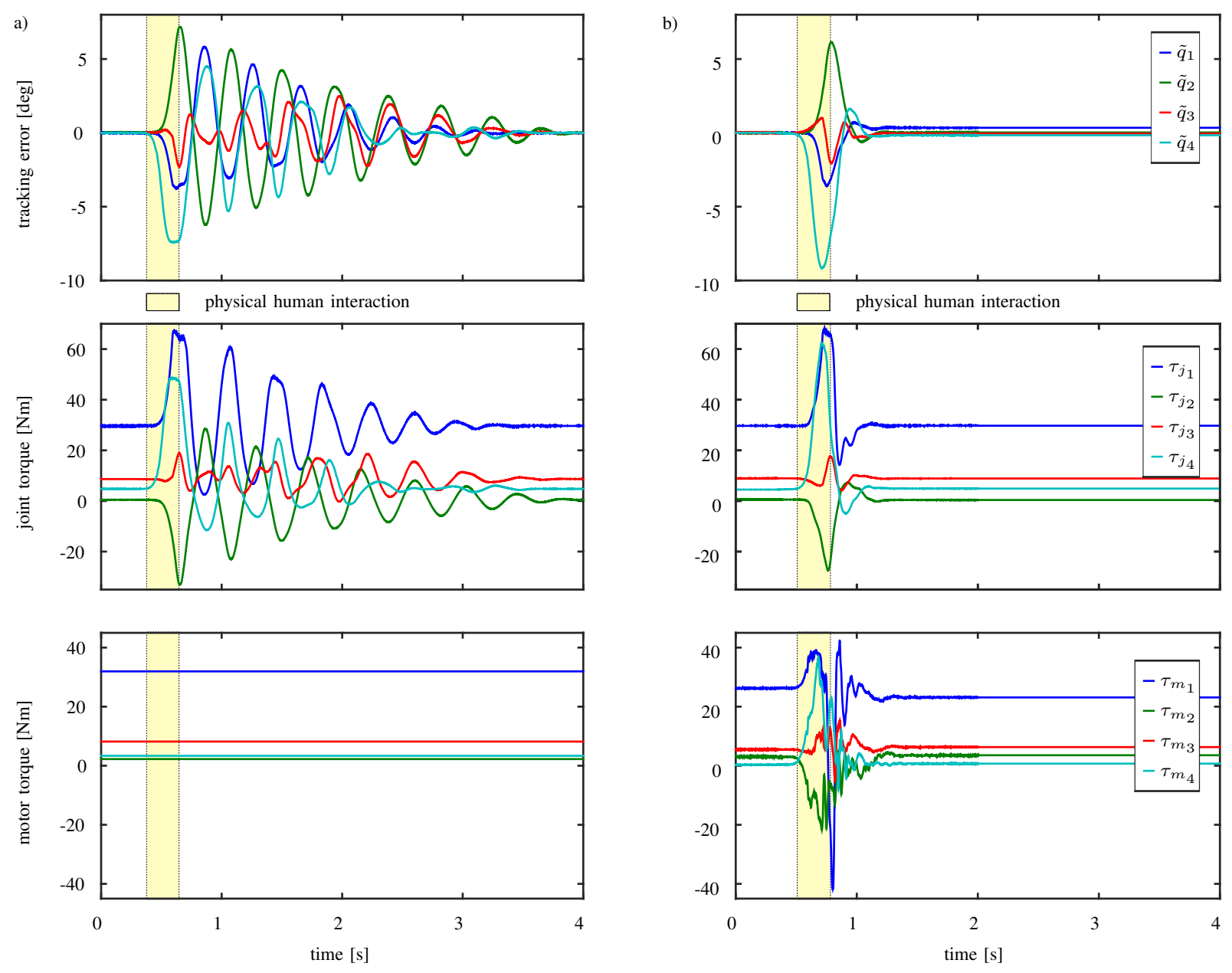

Fig. 5. Experimental result: damping performance after external disturbance through physical human interaction.
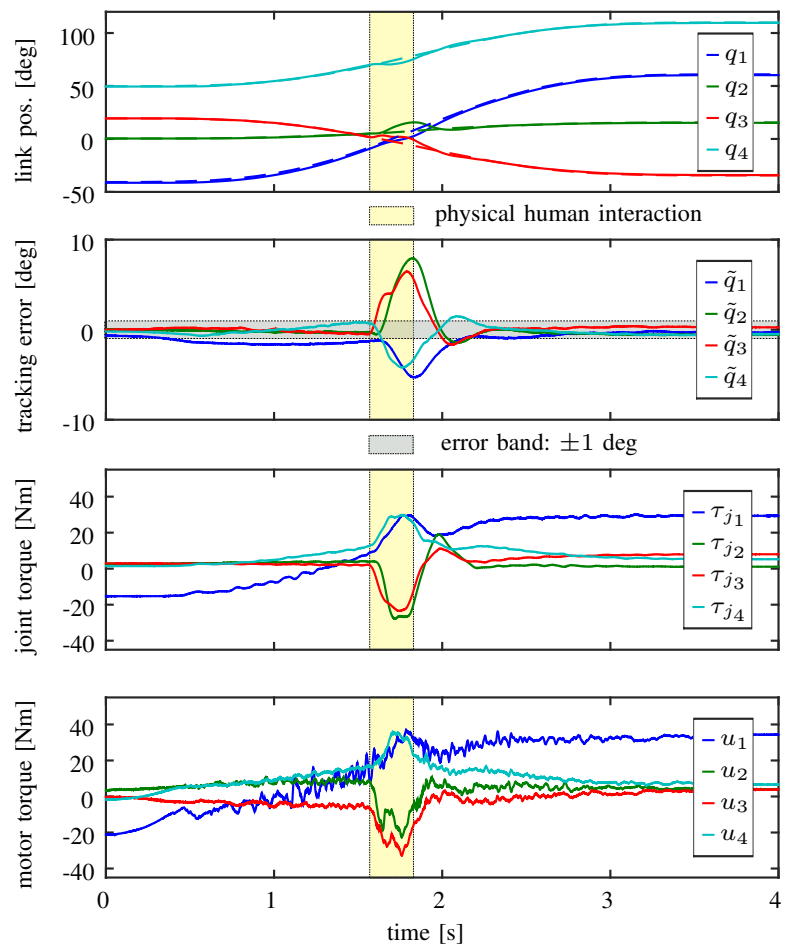

[21] G. Palli, C. Melchiorri, and A. De Luca, "On the feedback linearization of robots with variable joint stiffness," in IEEE Int. Conf. on Robotics and Automation, 2008, pp. 1753-1759.

[22] B. Brogliato, R. Ortega, and R. Lozano, "Global tracking controllers for flexible-joint manipulators: A comparative study," Automatica, vol. 31, no. 7, pp. 941-956, Jul. 1995. [Online]. Available: http://dx.doi.org/10.1016/0005-1098(94)00172-F

[23] S. Ozgoli and H. Taghirad, "A survey on the control of flexible joint robots," Asian Journal of Control, vol. 8, no. 4, pp. 332-344, 2006.

[24] A. van der Schaft, L2-gain and passivity techniques in nonlinear control. London New York: Springer, 2000.

[25] J.-J. Slotine and W. Li, Applied Nonlinear Control. Prentice Hall, 1991.

[26] F. Ghorbel, B. Srinivasan, and M. W. Spong, "On the uniform boundedness of the inertia matrix of serial robot manipulators," Journal of Robotic Systems, vol. 15, no. 1, 1998.

[27] S. Wolf, O. Eiberger, and G. Hirzinger, "The dlr fsj: Energy based design of a variable stiffness joint," in Robotics and Automation (ICRA), 2011 IEEE International Conference on, May 2011, pp. 5082 5089.

[28] R. A. Horn and C. R. Johnson, Matrix Analysis. Cambridge University Press, 2012.

[29] R. B. Nelson, "Simplified calculation of eigenvector derivatives," AIAA Journal, vol. 14, no. 9, pp. 1201-1205, sep 1976.

[30] M. I. Friswell, "Calculation of second and higher order eigenvector derivatives," Journal of Guidance, Control, and Dynamics, vol. 18, no. 4, pp. 919-921, jul 1995.

Fig. 6. Experimental results: the system is externally disturbed by physical human interaction while tracking a reference trajectory. 